\title{
Smart Home Automation System based on loT through Speech
}

\author{
Kajal Purwar \\ M.Tech Student \\ GGITS Jabalp
}

\author{
Ashok Verma \\ GGITS Jabalpur
}

\begin{abstract}
Home automation is becoming popular day by day due to numerous advantages. Till now the current development shows that the home automation system is managed by Emails, texts or some other applications. This paper provides an idea of using IOT for monitoring and controlling the home appliances via World Wide Web through wireless medium or Wi-Fi. The idea given in this paper aims at controlling home appliances through speech recognition by using raspberry pi as a server system and deploy in IBM Bluemix cloud platform so the system is accessible from anywhere and anytime. Raspberry pi known as a Single Board Computer (SBC) is proposed in this paper to achieve these goals. The voice command function will be given to control any appliances or devices at home. This will provide a better communication in automated home as compared to normal homes.
\end{abstract}

\section{Keywords}

Home Automation, Internet of Things, Speech Recognition, Smart Homes, Sensor System, Bluetooth, IBM Bluemix, Raspberry pi 3

\section{INTRODUCTION}

In this modern era, automation of everything is the need of the hourAutomation is the use of control systems and information technology to regulate equipment, industrial machinery, and processes, minimizing the need for human involvement. Automation plays an increasingly important role in the global economy and in day by day encounter. Engineers work to associate automated devices with mathematical and organizational tools to create complex systems for rapidly expanding parameters of applications and human activities. For the development of smart cities, there is a need to automate everything, so the concept of smart home automation system is an idea which is used to make the city smart. A Smart Home is one that provides comfort, security and gives the feeling of home to house members. Smart homes also provide Energy efficiency (low operating cost) and convenience at all times, for every individual at home.

Home automation means the monitoring and control of household objects intelligently for effective usage. The household objects should be intelligently interconnected as well as provide information for better operations. Home automation augmented with the Internet of Things (IoT) provides better flexibility in managing and controlling household objects in a wider aspect. This will support the interconnectivity of a large number of smart homes for better resource utilization in wider area.
People who are working are so busy so that they often forget to turn off their electrical devices when they are leaving the house for work. Those devices consume the electricity whole day so it leads to huge amount of electricity to go waste. So to overcome this problem Smart Home Automation System concept has been introduced.

"Smart Home" is a term used to characterize a residence house that has appliances, lighting, heating, cooling, security and camera systems that are capable of communicating with each other and can be remotely accessed from any room as well as remotely from any area by phone or internet through speech.

Internet of Things (IoT) is a new revolution of the Internet and the expansion of internet services. IoT digitizes our world, providing us with prolific amounts of data \& new delivery models that allow business to engage in new value creation special needs with a system that can respond to voice command and control all the appliances or devices in the home. The system should be cheap, easy to configure, easy to use and easy to run.

In this paper, Raspberry $\mathrm{Pi}$ is used which is installed in the home and all the appliances are attached with it through the relay. The recent developments in computers and other devices making them relatively less expensive than some time recently. It also leads to the development of smaller devices. Computer gets cheaper and smaller in size. Single Board computer (SBC) is a single circuit complete computer with Memory, Input/output (I/O) and different components of computers. Raspberry pi is an example of SBC that is popular and easily available in the market. It is a low-cost and small size computer used in Smart Home Automation System. RPi is a credit-card sized single board computer developed in the United Kingdom by the Raspberry pi foundation. There are various generations of Raspberry pi.

It also provides platform for communication between objects where objects can organize and manage themselves. It allows everyone to be connected anytime and anywhere. There are various technologies that enable IoT:

- $\quad$ RFID and near-field communication

- Optical tags and quick response codes

- Bluetooth low energy

- ZigBee

- Wi-Fi-Direct, etc.

The aim of the Wireless Home Automation System through voice mainly targets elderly and disabled person. 


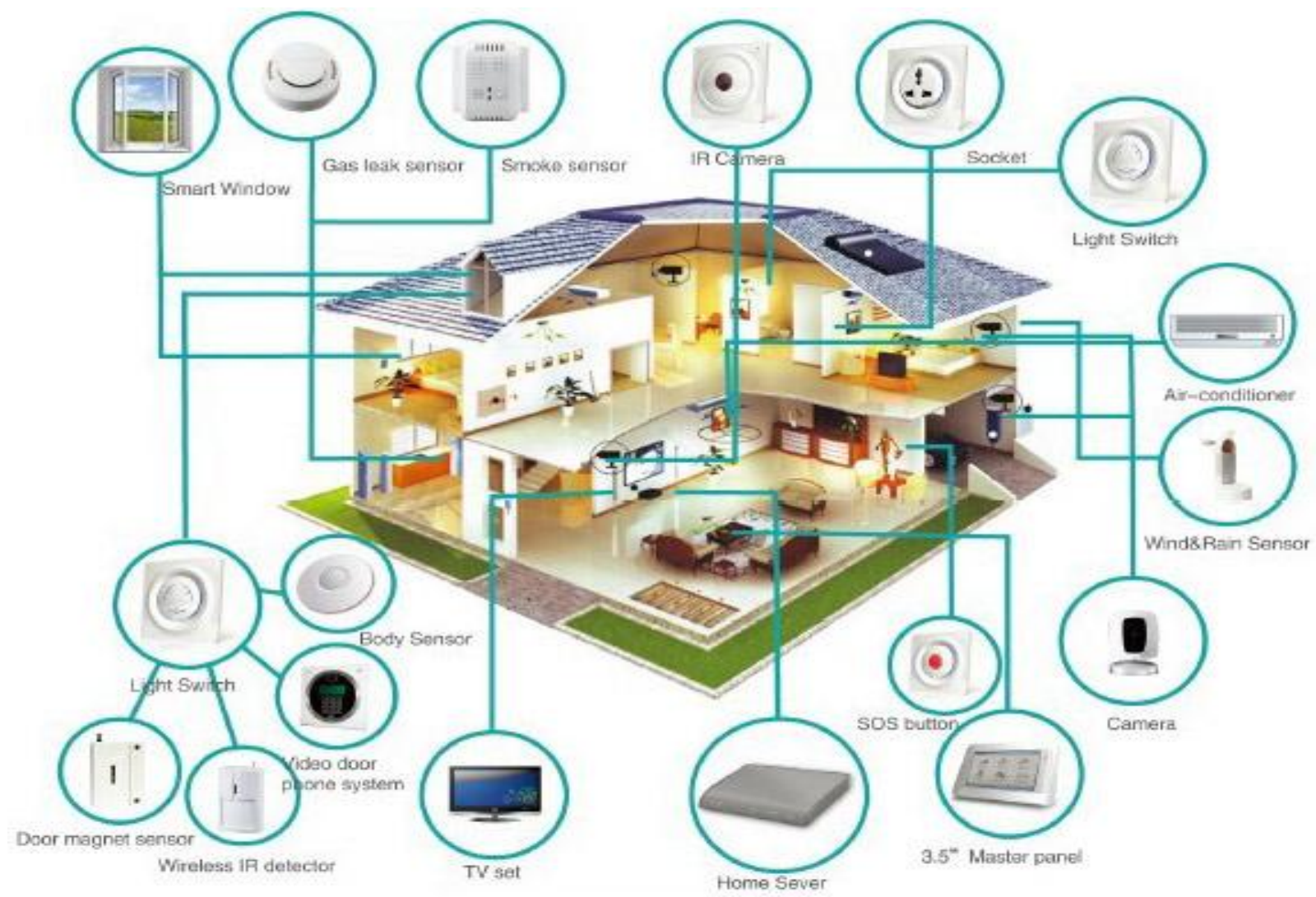

Fig 1: Smart Home using IoT [17] "http://smarthomeenergy.co.uk/sites/smarthomeenergy.co.uk/files/images/smarthome_0.jpg"

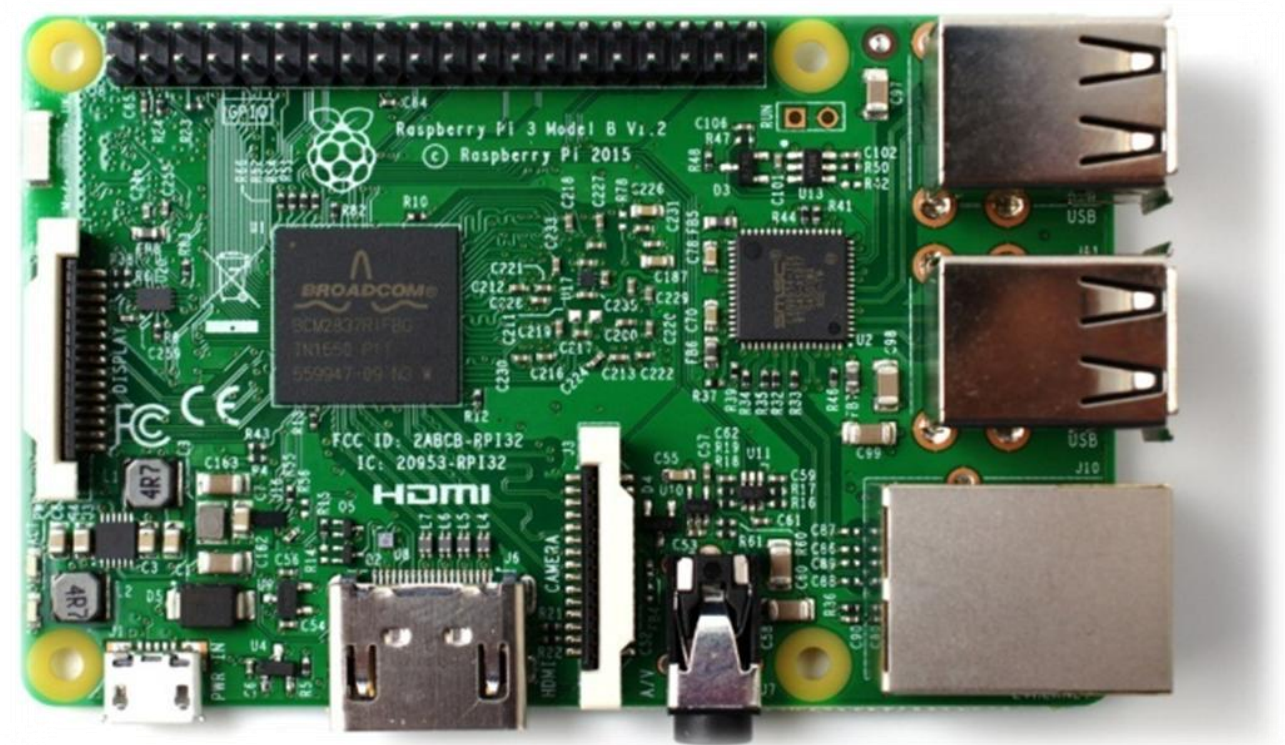

Fig 2: Raspberry Pi 3

In a Smart Home Automation System, the Raspberry Pi is combined with Cloud Computing to provide the communication between the person and the smart home. Cloud is a service provider or a type of internet based computing that provides shared computers processing resource and other devices on demand. Cloud provides services such as Infrastructure, Platform or Application.

IaaS (Infrastructure as a Service) provided to the consumer for processing, storage, network and other resources where the user is able to deploy and run their software which can include operating system and applications.

PaaS (Platform as a Service) is a cloud computing service that provides a platform to the user to deploy, run and manage applications which are created using the programming languages, services which are supported by the user.

SaaS (Software as a Service) provided to user to use the provider's application running on a cloud infrastructure. 
The cloud platform is used in this project is IBM Bluemix.

The developer using Bluemix for:

- To quickly bring products and services to market at lower cost.

- Continuously grant new functionality to their applications.

- To extend existing investment in IT infrastructure.

IBM Blue mix is an open standard cloud based platform as a service developed by IBM and supports several languages, services as well as integrated DevOps to build, run, deploy and manage applications on the cloud. Bluemix depends on Cloud Foundry open technology and keeps running on Soft Layer infrastructure. Bluemix supports several programming languages including Java, Node.js, PHP, Swift, Python, Ruby, etc.

Node-RED is a tool for wiring hardware devices, service and API's together in interesting ways as a part of IoT. It alsogives a browser-based editor that makes it simple to wire together flows using the wide range of nodes in the palette that can be sent to its runtime in a single-click.

The rest of the paper organized as follows: Section II provides a Literature Review of the system. Section III discusses the Methodology of the system. Section IV discuss about the Proposed System. Section V contains result Analysis of the System. Section VI contains Conclusion and the future research to make the system more effective.

\section{LITERATURE REVIEW}

In the existing system, the studies on Home Automation System focuses on addressing the problems or power consumption range of operation and cost of the whole system. To automate the appliances, various methods are used like SMS and Email. The work presented here is focused on fast and easily accessible of a wireless smart home automation system to reduce the manual work and everyone accesses this system. It is having low cost, secure, and access fast as compared to the previous systems.

Ana et al. [1] proposed the work on Home Automation System through Siri enabled mobile devices but the Siri application is installed on the iOS device then only it is accessible to users. They used Apple's Siri for the efficient speech recognition module which is used for translating voice commands and sending it to the actuator of the system through Zigbee networks. The limitation of the work is that there is no utilization of sensors which shows the current status of the appliances and for each appliance, it requires module so it makes the system uneconomical and limited.

Piyare \& Tazil [2] et al. proposed the work on this by using the Bluetooth technology for Home Automation System using an Arduino board as well as the wireless system. Through a Bluetooth connection, a cell phone or mobile device is used to send commands to the Bluetooth antenna of the Arduino board but the disadvantage in this is that it is applicable only for the short distance.

H. Alshueili et al. [3] proposed a work of Voice Recognition Based Wireless Home Automation System by using Zig-Bee transceivers. It used DPCM (Differential Pulse Code Modulation) and A/D converter to convert the voice command into digital form then pass through the microcontroller to analyze or produce string commands and then it will execute that command.
S. Hidayat et al. [4] proposed a work on Home Automation system through Voice schedule. In this paper it proposed a system which is using the Raspberry Pi with the devices attached in a Relay and by using the voice command it will control the devices but the disadvantage of this work is that the wired medium is used so the cost of the system increases and the scheduling system is activated approximately 1 minute after the raspberry pi is activated.

S. Jain et al. [5] proposed a work on Raspberry Pi based Interactive Home Automation System through E-mail in which it design a basic home automation application on Raspberry Pi through reading the subject on Email and it used Python programming language which is a default programming environment provided by the Raspberry Pi. It used LED's to indicate the switching actions. The limitation of this work is called tariff in home automation through DTMF (Dual Tone Multi Frequency) and it doesn't provide any security for the system in case of Email is hacked then any unauthorized person can access.

M. Narendar et al. [6] proposed a work on Raspberry Pi based Advanced Scheduled Home Automation System through Email but the automation process that is employed in this work in scheduled automation. The limitation of this work is it used the Raspberry Pi 2 model B so that less no. of devices are connected with it and more that one instruction was given by the E-mail than one instruction interrupts the other instructions.

D. Pavithra et al. [7] proposed a work on IoT based Monitoring and Control System for Home Automation and it used the portable devices for the user interface. They can communicate with Internet gateway, by using low power communication protocols like ZigBee, WI-Fi, etc. It makes web portal to interact with the devices at home and web portal is accessed via Smartphone. It doesn't provide security to their system and limited no. of devices are used in their work.

B. Pandya et al. [8] proposed a work on Android Based Home Automation System using Bluetooth \& voice command. It used an Arduino microcontroller for connecting the appliances, a Bluetooth module for transferring the signal which is running on Smartphone an Android application. The limitation of this work is that it uses a microcontroller which converts the byte signal into string format so there must be a delay in the system.

To resolve this problem, Wi-Fi technology can be used for a wider range of applications. So in this paper, Raspberry Pi 3 is used to resolve the problem and the number of Input/output ports in the Raspberry Pi also increases so we can attach more than 5 appliances to it.

\section{METHODOLOGY}

The Smart Home Automation System is an integrated framework to facilitate people with an easy to use home automation system that can be fully operated based on voice/ speech commands. The system is constructed in a way that it is easy to install, configure, run and maintain by any type of people.

The system is operated as the voice command is given through a microphone/cell phone so that the raspberry pi is performing an action on the basis of voice commands. The speech is mapped through commands by using the Alexa Voice Service develops by the Amazon.

The Alexa Voice Service (AVS) enables outsiders to add intelligent voice control to any associated product that has a 
microphone and speaker. Users can ask Alexa to play answer questions, order dinner, get news and more on voice enabled product like the home intercom. Voice is the future and providing a natural interface to make a complex thing simple.

Steps of Alexa Voice service:

- Say to Echo" Alexa: discover my device" it will take few seconds to find the new device. If, it doesn't find it, try killing and restart the process.

- Now, Say "Alexa: turn off device", or "Alexa, device on". It will respond according to the commands through Echo IP Address.

The front end is designed by using the node red.

Node-red is a software tool developed by IBM for wiring together hardware device, APIs, and online services as part of the Internet of Things. The runtime is formed on Node.js. The movements are saved using JSON.

In this system, no. of devices is getting attached to it and for the security of home camera is also get attached in front of the door and access those camera records from anywhere in the cell phones.

The wireless smart home automation system that will control appliances in a home from anywhere in the world. It will also allow user to view data from the PIR motion sensor via the internet to detect intruders. This work will be utilizing platforms like the IBM's IoTF (Internet of Things Foundation), Raspberry Pi 3 and Bluemix packages.

\section{PRPOPOSED SYSTEM}

The system is divided into two parts:

\subsection{Sending commands to the Raspberry Pi}

The server side script running on laptop or on a web server that takes input commands from the user and correspondingly sends it to the client (Raspberry Pi). Here, we will be utilizing commands to turn a light ON/OFF. When we pass the command to turn $\mathrm{ON}$ a light through the server side script, the data is relayed to the Raspberry Pi and its GPIO pin turns ON a relay. The system also forwards state updates to the server on whether the light is ON/OFF.

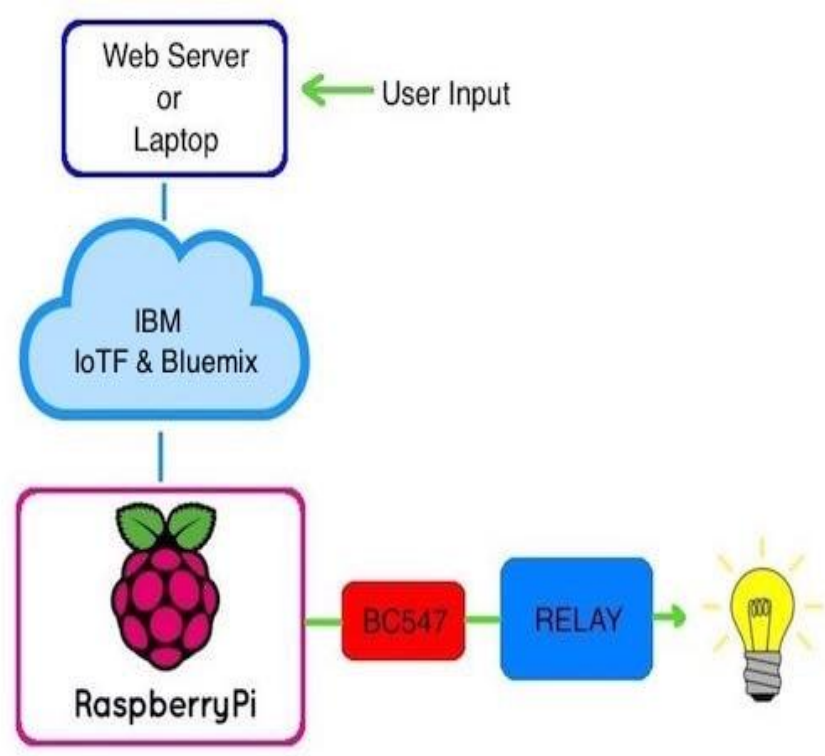

Fig 3: Sending commands to Raspberry pi

\subsection{Receiving data from Raspberry $\mathrm{Pi}$}

- When data is sending from PIR motion sensor to the Raspberry $\mathrm{Pi}$, a script is run which reads the sensor through the GPIO pins and broadcasts the data through IoTF platform.

- This can be viewed through the IoTF console or a custom web application designed utilizing the platform.

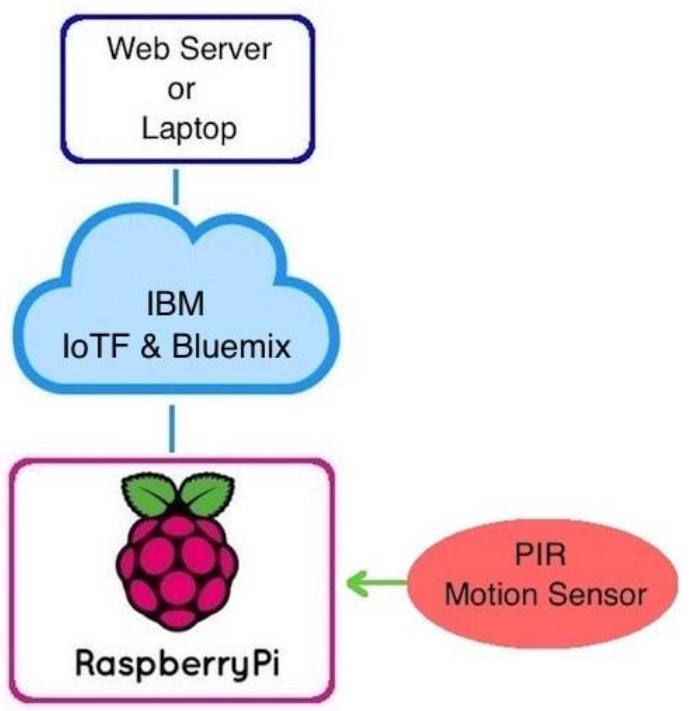

Fig. 4: Receiving commands from Raspberry pi

The Smart Home Automation system relies on the cloud for speech to command conversion because the system is deployed on the cloud platform and the platform is an open standard, free and can be accessed from any location.

The hardware design of the home automation system consists of Relay along with raspberry pi circuit board. The appliances are connected to the Relay and relay connected with Raspberry Pi 3 . The appliances are of any type.

Hardware needed in the home automation system is:

- $\quad$ Raspberry Pi 3

- Microphone

- Relay

- Laptop

- Devices

Raspberry Pi 3 comes with GPIO pin that serves as a connector to electronic devices. It acts as a central processing unit for the whole system. Raspberry Pi 3 is not equipped with an audio input through wires. It is equipped with a cell phone through a wireless medium. The cell phone is interacting with the devices through $\mathrm{Wi}-\mathrm{Fi}$ which is an additional feature of Raspberry Pi 3.

The software design of the system focuses on how to control the system through a voice that would enable home devices. The system is accessed from the cloud and there will be no delay in the service because nowadays $4 \mathrm{G}$ networks are used and it is very fast so there is no delay in the system. The $4 \mathrm{G}$ network offers high-speed mobile internet connection and the quality of $4 \mathrm{G}$ is VoLTE (Voice over Long Term Evolution). The delay is calculated by the Apache JMeter which is open source software and a pure Java application designed to load test functional behavior and measure performance. It is 
utilized to test performance both on static and dynamic resources.

The proposed home automation system is an integrated system to facilitate elderly and disabled people with an easy to use that can be fully operated by voice commands. It is portable, easy to install, configure, run and maintain. The system is in Automatic mode. In the installed when the user wants to function automatically, first the voice command saying "Alexa" then the Alexa voice service has activated.

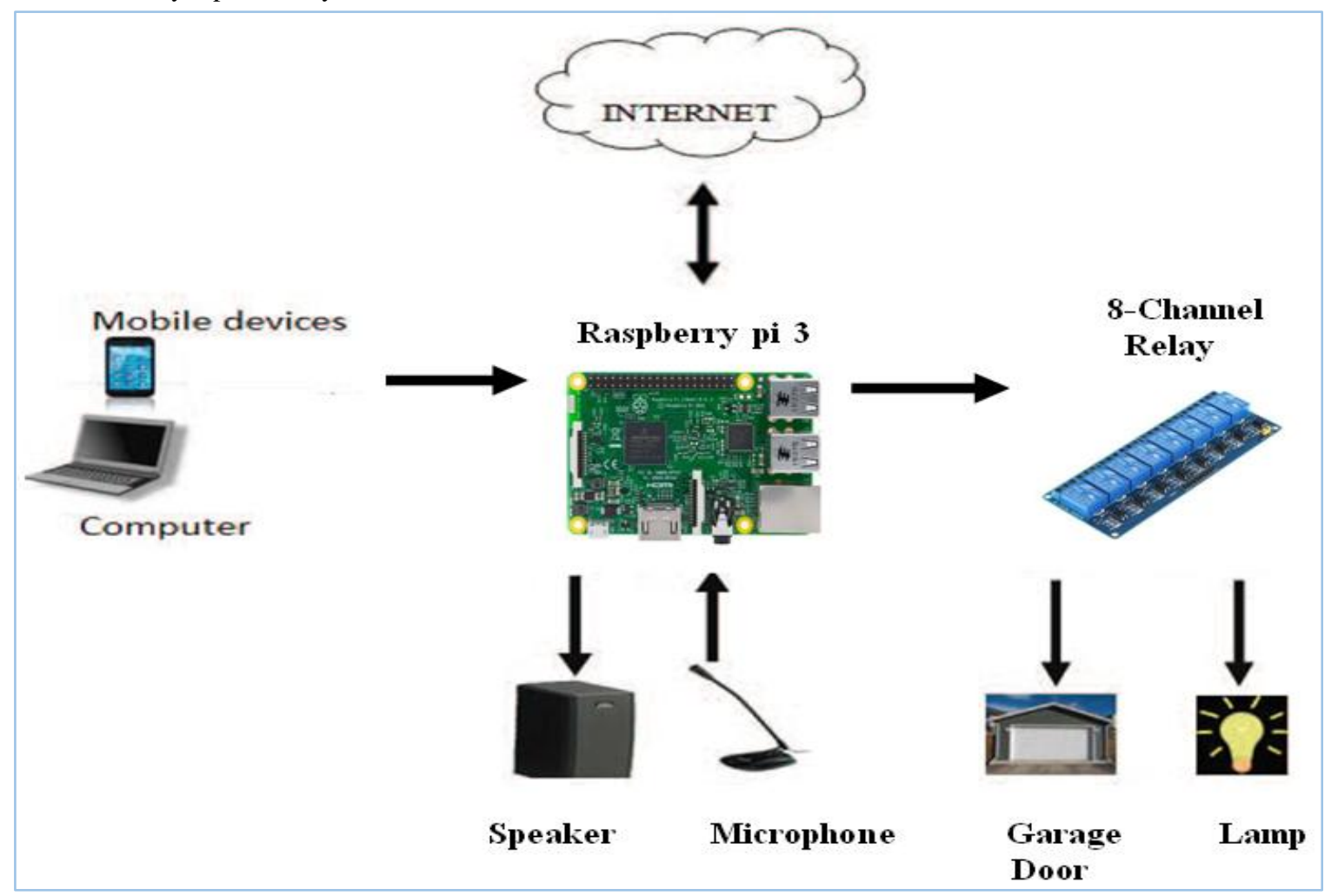

Fig 5: Overall System Design

\subsection{Block Diagram of ProposedSystem System}

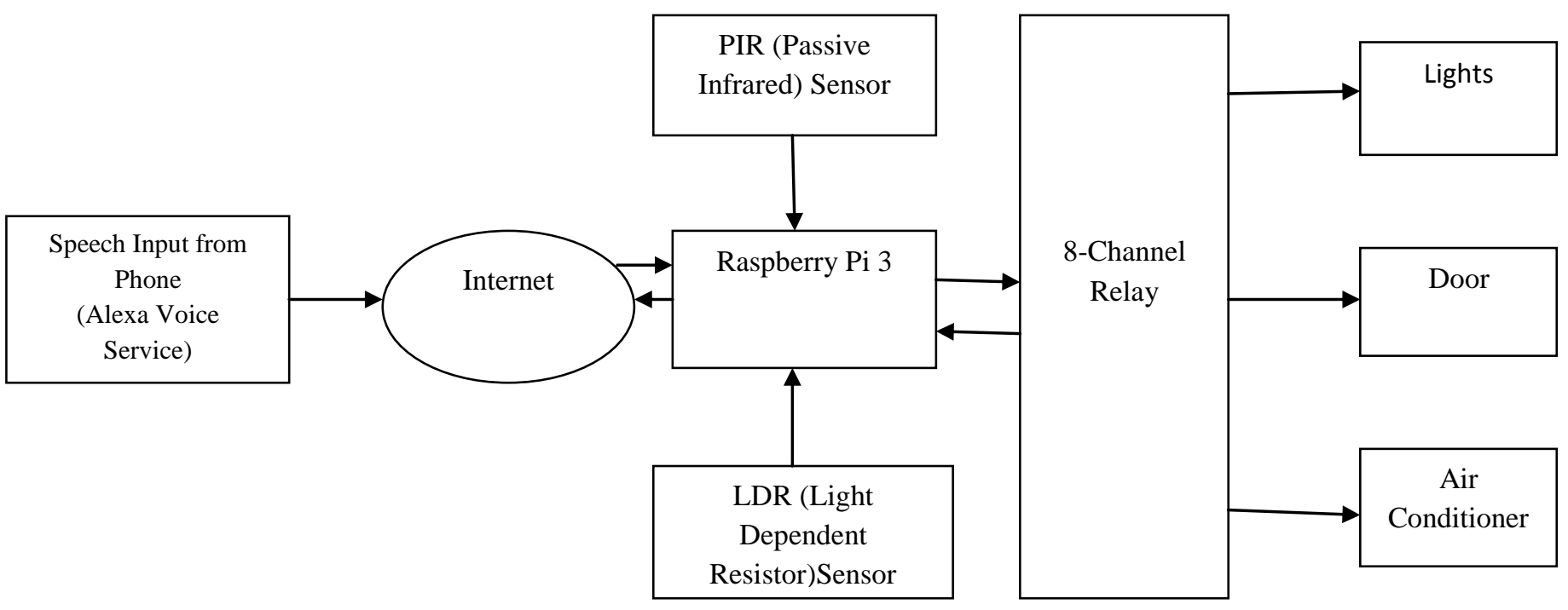

Fig 6:Flowchart of the System 


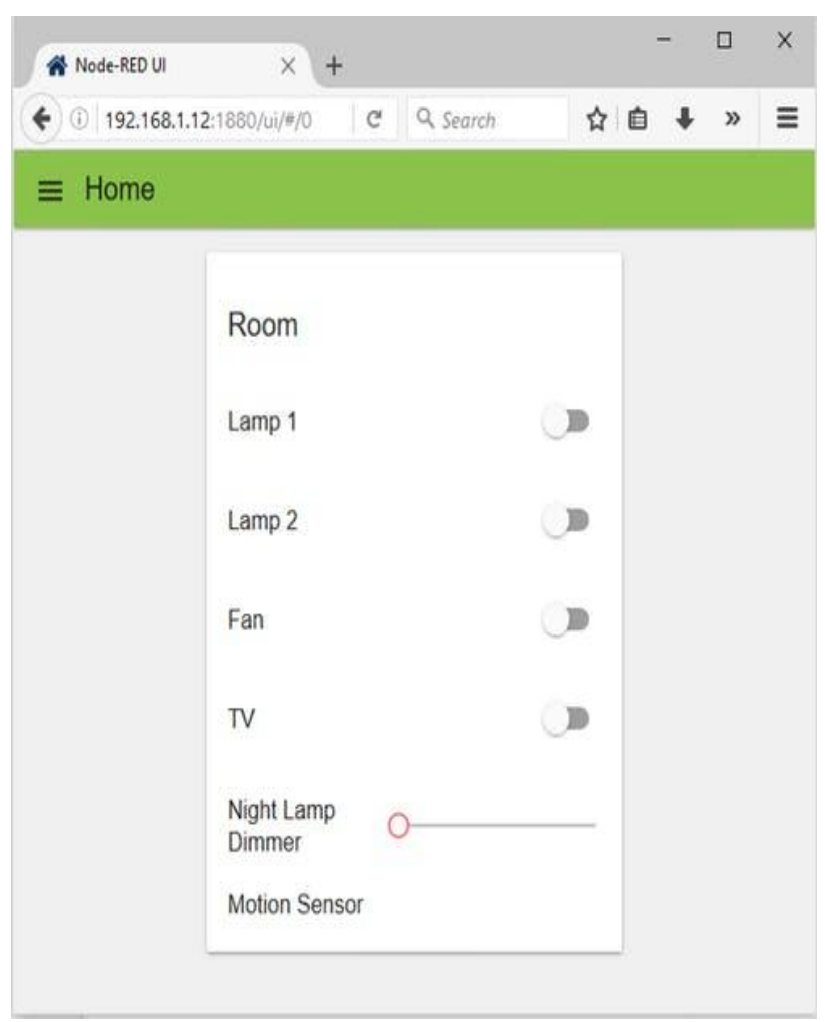

Fig 7: Graphical User Interface (GUI) coded in Node-Red



Fig 8: Connection of devices with Raspberry Pi 3



Fig 9: Lamp ON

With the above result, we can say that the system working properly and delay are less as compared to the previous systems. The delay is calculated by using the Apache JMeter.

The elderly and disabled people can easily use this system. If the sensor is used for all the appliances, so it is very easy to see the state of appliances and according to that, we can give the voice command to the system. In the system, there is no need of any type of voice-to-text-converter to convert the voice messages into command.

We can directly give the voice command to the system and it works in less time. Because of this, the efficiency of the system increases and it is easy to configure, easy to install \& easy to use. We are also providing power backup for the smooth and continuous functioning of the system in case of power failure. The power bank is used to charge the Raspberry Pi so there is less chance to slow down the system. Any type of person can use the system and it makes the system very cheap so everyone can afford it very easily. There is no need of any additional storage for this to store any data because it can access the system through the cloud.

\section{RESULT ANALYSIS}

In order to demonstrate the proposed system, we are comparing our system with the previous system according to the user specifications and technical specifications.

The Table 1\& Table 2 shows the comparison of proposed system with the previous system on the basis of some parameter according to the user specifications and technical specification respectively. 
Table 1. Relevance of Proposed System as per User Specification

\begin{tabular}{|c|c|c|c|}
\hline S No. & User Oriented Metric & $\begin{array}{c}\text { Earlier } \\
\text { Method }\end{array}$ & $\begin{array}{c}\text { Proposed } \\
\text { Approach }\end{array}$ \\
\hline 1. & Ease of Use & Last & First \\
\hline 2. & Simplicity & Similar & Very well \\
\hline 3. & Resource Requirements & More & Less \\
\hline 4. & Speed & Low & High \\
\hline 5. & Understand ability & Complex & Moderate \\
\hline 6. & Security & Less & Moderate \\
\hline
\end{tabular}

Table 2. Relevance of Proposed System as per Technical Specification

\begin{tabular}{|c|c|c|c|}
\hline S No. & $\begin{array}{c}\text { Technical } \\
\text { Specification }\end{array}$ & $\begin{array}{c}\text { Earlier } \\
\text { Method }\end{array}$ & $\begin{array}{c}\text { Proposed } \\
\text { Approach }\end{array}$ \\
\hline 1. & Hardware Complexity & Complex & Moderate \\
\hline 2. & Code Complexity & Complex & Moderate \\
\hline 3. & Delay & High & Low \\
\hline 4. & Flexibility & $\begin{array}{c}\text { Very } \\
\text { Limited }\end{array}$ & Available \\
\hline 5. & Reliability & Moderate & High \\
\hline 6. & Cost & Very Costly & Less \\
\hline 7. & Portability & No & Yes \\
\hline
\end{tabular}

\section{CONCLUSIONS}

The Smart Home Automation system is implemented by using the Raspberry Pi that automates the no. of appliances through the use of voice/ speech recognition capability. The speech to command convertor is used to give the command to the devices. The Raspberry Pi's network and DNS settings, using the Raspbian operating system are configured with the mobile devices. The system is able to automate the appliances at home. There are many advantages of Raspberry pi as compare to other devices are: It is robust, and the ability to run multiple programs. The technology behind this is a benefit for the mankind. The system is fully functional and controlled through the wireless system.

\subsection{Future Work}

The future scope of the system is to make the system secured and everyone access from any place.

\section{REFERENCES}

[1] Marie, A., Benedict, I., Zandrae, A., Neil, A., Gustilo,R. 2015 Home Automation Using Raspberry Pi through Siri Enabled Mobile Devices.

[2] Piyare,R., and Tazil,M. 2011 Bluetooth based Home Automation System using Cell Phone.

[3] Alshueili, H., Gupta,G., Mukhopadhyay,S. 2011 Voice Recognition Based Wireless Home Automation System.

[4] Hidayat, S., Firmanda, S. 2015 Scheduler and Voice Recognition on Home Automation Control System.

[5] Jain, S., Vaibhav, A., Goyal,L. 2014 Raspberry Pi based Interactive Home Automation System through E-mail.

[6] M. Narender, M. Vijaylakshmi, "Raspberry Pi based Advanced Scheduled Home Automation System through E-mail", IEEE International Conference on Computational Intelligence and Computing Research, pp: $1-4,2014$

[7] D. Pavithra, R. Balakrishnan, "IoT based Monitoring and Control System for Home Automation", Global Conference on Communication Technologies (GCCT), pp: 169-173, 2015

[8] B. Pandya, M. Mehta, N. Jain, "Android Based Home Automation System Using Bluetooth \& Voice Command", International Research Journal of Engineering and Technology (IRJET), pp: 609-611, Volume: 03 Issue: 03, 2016

[9] A. Ramya, T. Srihari, "Raspberry Pi (Model B) Based Interactive Home Automation System", International Journal of Trend in Research and Development, Volume 3(1), pp: 1-4, 2016

[10] P. Hazari, A. Andurkar, "System for Voice and Facial Recognition using Raspberry Pi", International Journal of Advanced Research in Computer and Communication Engineering Vol. 4, Issue 4, pp: 438-440, April 2015.

[11] Digvijay S. Nikam, Vinayak R. Shinde, Swapnil N. Yavalkar, Nikhil B. Mate, "A Review on Cloud Based Automation System", International Journal of Innovative Research in Computer and Communication Engineering, Vol. 3, Issue 9, September 2015.

[12] D. Sunehra and M. Veena, "Implementation of Interactive Home Automation System Based on Email and Bluetooth Technologies", International Conference on Information Processing (ICIP), 13 June 2016. 